\title{
Point-of-care pleural effusion ultrasound in critically ill patients
}

\author{
R. García Álvarez¹, C. Pinilla Silva², N. Zurera Plaza1', L. Alvaro-Gracia Heredero1, D. \\ Benguria Plaza ${ }^{1}$, F. Pérez-Cerdá Silvestre ${ }^{1}$ \\ ${ }_{1}^{1}$ Department of Anesthesiology and Intensive Care. Universitary Hospital 12 October - \\ Madrid (Spain), ${ }^{2}$ Hospital Infanta Cristina - Badajoz (Spain).
}

Background and Goal of Study: Point-of-care ultrasound (PoCUS) is increasingly being used by the physicians to complement the findings of physical examination in the intensive care units (ICU). PoCUS lung is non-invasive, devoid of radiation exposure and can be performed rapidly and repeatedly as needed at bedside. It is a more sensitive means than both chest radiography and computed tomography for identifying pleural effusions and can detect accumulations as low as 10 $\mathrm{mL}$. We describe the way of detecting and measuring and two different clinic cases.

Materials and Methods:Pleural effusion is best detected by placing a curvilinear or phased array probe in the midaxillary line, yielding an image in the coronal plane.

The liver or spleen and diaphragm should be identified.

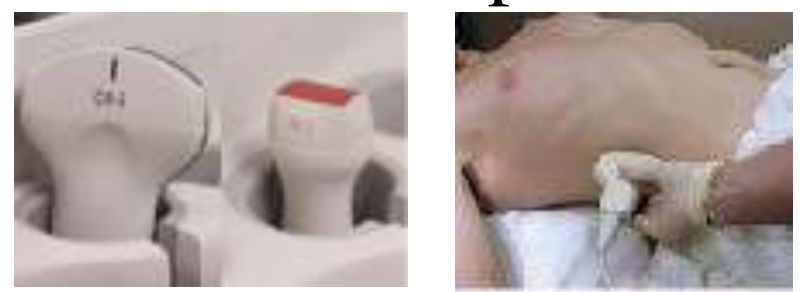

Results and Discussion:When present, fluid will be seen as an anechoic area superior to the diaphragm . With larger amounts of fluid, atelectatic lung tissue may be seen moving within the anechoic fluid (figure 1).

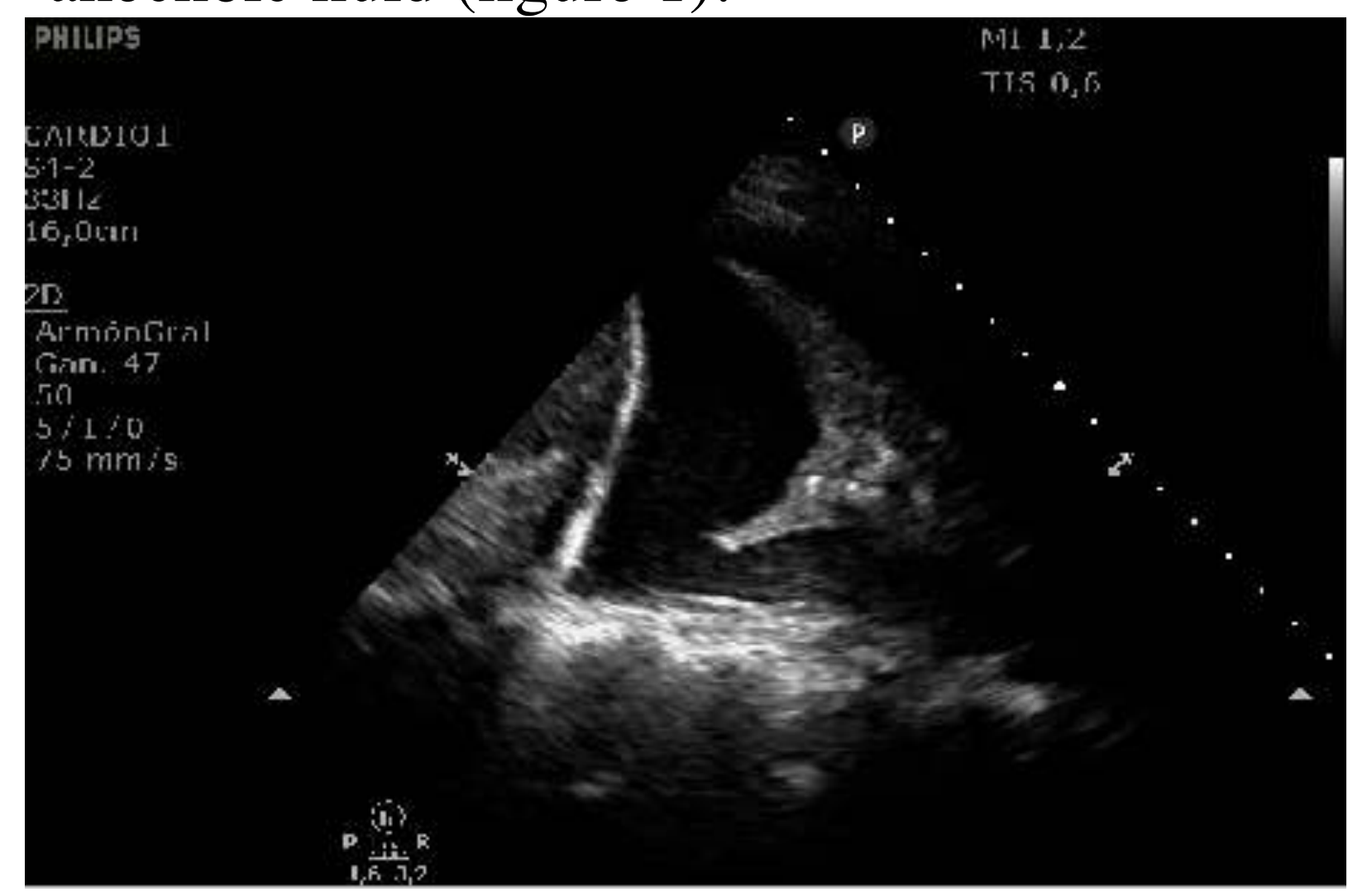

Figure 1

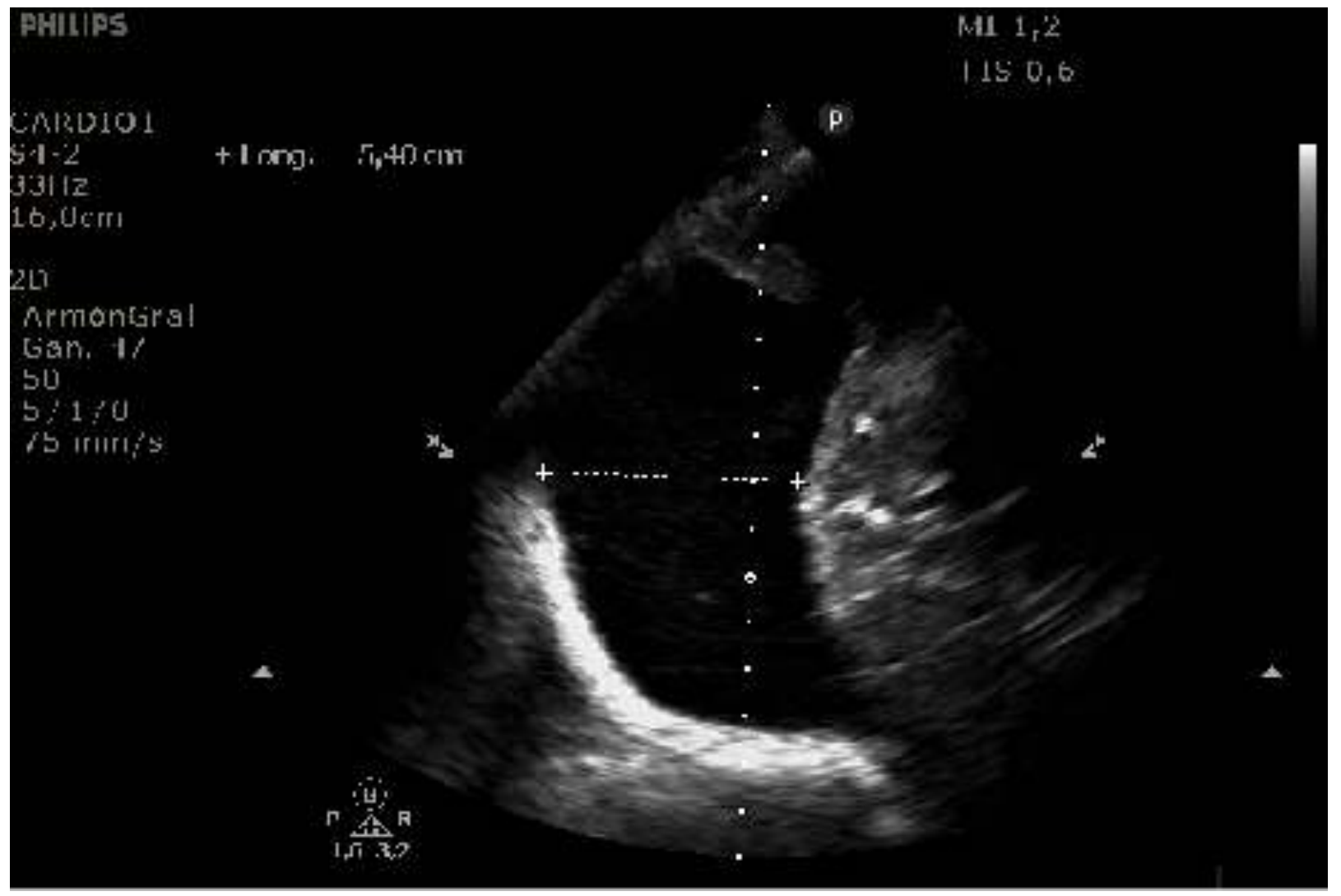

Figure 2

Ultrasonography can be used to quantify the amount of fluid (figure 2) with the probe turns in transverse plane: we measure the distance between lung and posterior chest wall; if this distance is superior to $30 \mathrm{~mm}$, the predictive volume is more $\tan 500 \mathrm{ml}$. There is a published formula effusion volume $(\mathrm{ml})=20 \times$ distance between lung and posterior chest wall $(\mathrm{mm})$ but we believe that knowledge of the exact amount of fluid has limited usefulness in clinical practice, we prefer a qualitative approach.

Conclusion:PoCUS may represent an accurate diagnostic approach to the patient with pleural effusion in the ICU with the probe in coronal plane, and when the effusion is detected, it must be turned to transverse view for measure. PoCUS lung helps in deciding thoracentesis and it provides visual guidance for it and if we don't do it, we can measure effusion everyday and see the evolution.

\section{Bibliography:}

1. Balik M et al.Ultrasound estimation of volume of pleural fluid in mechanically ventilated patients. Intensive Care Med 2006; 32: 318-321.

2. Remérand F et al. Multiplane ultrasound approach to quantify pleural effusion at the bedside. Intensive Care Med 2010; 36 : 656-664. 